\title{
Laparoscopic Gastric Plication in Obese Egyptian Patients: Technique and Initial Short-term Outcome
}

\author{
Ahmed A EIGeidie ${ }^{1 *}$, and Nabil Gad ElHak ${ }^{2}$ \\ ${ }^{1}$ Gastroenterology Surgical Center, Mansoura University, Mansoura, Egypt \\ ${ }^{2}$ Gastroenterology Surgical Center, Jehan st., Mansoura, Dakahlia, Egypt
}

\begin{abstract}
Purposes: Laparoscopic gastric plication (LGP) is a new restrictive bariatric procedure that emerged to avoid the problems and to reduce the cost of more popular restrictive procedures particularly laparoscopic sleeve gastrectomy. In this study we present the surgical technique and initial short-term outcome of LGP for treating morbidly obese Egyptian patients.
\end{abstract}

Materials and methods: Data of all patients who underwent LGP at the Gastroenterology Surgical Center, Mansoura University, Egypt between March 2010 and November 2012 was retrospectively reviewed. Early cases were done by the original two-bite technique while later cases were plicated using four-bite stitches.

Results: Data of 47 patients (9 males and 38 females) who had completed their 3 - and 6-month postoperative follow-up visits were analyzed. The mean age was 36.8 years. Mean follow-up was 8.6 months. Mean preoperative weight was $109.4 \mathrm{~kg}$ with a mean BMI of $40.7 \mathrm{~kg} / \mathrm{m}^{2}$. All the operations were completed laparoscopically. Mean operative time was $85 \mathrm{~min}$. Mean hospital stay was 1.8 days. At 3 months following LGP, mean weight loss was 24.6 $\mathrm{kg}$, and at 6 months $36.4 \mathrm{~kg}$. The mean \%EWL was 41.4 and 50.5 at 3 and 6 months respectively. Two out of 58 patients (3.4\%) developed prolonged nausea, vomiting and sialorrhea for 5 and 7 days but subsided subsequently with medications. No patient required reoperation or readmission.

Conclusions: LGP is a safe, feasible and effective weight reducing surgery that suits most of our Egyptian patients as an alternative restrictive procedure.

Keywords: Bariatric; Laparoscopic; Gastric plication; Egyptian

\section{Introduction/Purposes}

Laparoscopic gastric plication (LGP) is a newly emerging restrictive bariatric operation. In LGP, the greater curve of stomach is sutured vertically in rows to reduce its volume to help obese patients to consume less food with early satiety similar to other restrictive procedures such as laparoscopic sleeve gastrectomy (LSG) and laparoscopic adjustable gastric banding (LAGB). The operation was initially proposed by Talapour et al. as an alternative to LSG in order to avoid its potentially serious complications such as bleeding and staple line leakage [1]. Their results were impressive and had been shown to provide meaningful weight loss with acceptable morbidities. Subsequently, several authors had demonstrated that the operation is feasible and safe with comparable early results to other types of conventional procedures [2-8].

In Egypt, restrictive procedures such as LSG and LAGB are the most popular procedures whereas, more invasive operations such as gastric bypass and biliopancreatic diversion are generally reserved as a second-stage intervention. The potential advantages of LGP over other types of bariatric surgery are apparent. LGP does not require expensive staplers used for sectioning the stomach (as in LSG) and it does not require foreign body implantation with its complications (as in LAGB). The cost is obviously lower than other existing procedures making LGP a preferable option for obese patients in developing countries including Egypt.

The most popular surgery we performed for morbidly obese patients was LSG. Many of our Egyptian patients could not afford the high cost of LSG. Moreover, in Egypt the expenses of bariatric surgery are not covered by the health insurance as bariatric surgery is considered a cosmetic one and the patients have to pay its cost. For this reason we started to offer LGP as an alternative to LSG for those patients who cannot afford the cost and expenses of LSG.
Reports regarding LGP are scarce worldwide and we have few data of LGP in Egyptian patients. In this study, we are reporting the surgical technique and the short-term outcome of LGP as an alternative option for management of morbidly obese Egyptian patients in a single center.

\section{Materials and Methods}

At our hospital we started laparoscopic bariatric surgery since 2009. The data of morbidly obese patients who were underwent LGP were prospectively analyzed. The first 15 cases of LGP (representing our learning curve) were not included in our analysis. An informed consent was obtained from all patients after explaining the benefits and risks, and our local ethical committee approved the study.

Data of all patients who underwent LGP for the treatment of morbid obesity at the Gastroenterology Surgical Center, Mansoura University, Egypt between March 2010 and January 2013 were retrospectively reviewed from our database. Only the data of patients who had completed their 3-and 6-month follow-up visits at the time of the study were further analyzed. Data collected included demographics, operative time, length of stay, postoperative complications, cure of comorbities and weight loss. Weight loss was expressed as the percentage of excess weight loss (\%EWL). The ideal weight was derived

*Corresponding author: Ahmed A EIGeidie, Gastroenterology Surgical Center Jehan st., Mansoura, Dakahlia, 35516 Egypt, Tel: +201005290111; E-mail: drarelgeidie68@hotmail.com

Received August 10, 2013; Accepted November 21, 2013; Published November 27,2013

Citation: EIGeidie AA, EIHak NG (2013) Laparoscopic Gastric Plication in Obese Egyptian Patients: Technique and Initial Short-term Outcome. Surgery 3: 151 doi:10.4172/2161-1076.1000151

Copyright: (c) 2013 EIGeidie AA, et al. This is an open-access article distributed under the terms of the Creative Commons Attribution License, which permits unrestricted use, distribution, and reproduction in any medium, provided the original author and source are credited. 
from the Metropolitan height and weight tables using the mid-weight for a medium-frame [9].

The US National Institute of Health criteria for bariatric surgery were used for patient selection [10]; patients required a body mass index (BMI) of $>40 \mathrm{~kg} / \mathrm{m}^{2}$ or $>35 \mathrm{~kg} / \mathrm{m}^{2}$ with at least one comorbidity and an absence of psychological conditions that influence his/her perception of the study protocol and postoperative evaluations and recommendations. Patients with extreme of age $(<18$ and $>65)$ were excluded. We required all patients to be included had attempted nonsurgical weight loss treatment in the past with history of obesity of more than 5 years. All patients were informed extensively regarding the potential benefits, complications and alternatives prior to the operation. Preoperatively, all patients underwent upper GI endoscopy, barium study, blood tests, and abdominal ultrasound. Anticoagulants were given $12 \mathrm{~h}$ preoperatively, and chemoprophylaxis with antibiotics was given with the induction of anesthesia.

\section{Surgical technique}

The procedure was performed in French position with five trocars at upper abdomen with the patient in a $30^{\circ}$ reverse Trendelenburg position similar to LSG (Figure 1). Two 10-mm ports were used; one was $20 \mathrm{~mm}$ below the xiphoid process and slightly to the left of the midline for the camera and one at right midclavicular line for the left surgeon's hand. Three 5-mm ports were used; one located just below the xiphoid process for liver retraction using a regular $5-\mathrm{mm}$ instrument, one at the left anterior axillary line just below the costal margin for the assistant instrument, and one at the left midclavicular line for the surgeon's right hand.

Closed pneumoperitoneum of 16-18 $\mathrm{mmHg}$ was achieved using Veress needle through the umbilicus or upper left quadrant. Using the Harmonic ACE (Ethicon Endo-Surgery, Cincinnati, Ohio), the omentum and the gastrepiploic vessels were dissected away from the greater curvature, starting 3-4 cm from the antrum till $2 \mathrm{~cm}$ below the angle of His. During greater curvature mobilization, it was important to stay $1-2 \mathrm{~cm}$ away from the stomach to avoid thermal injury to the stomach and subsequent risk of leak (Figure 2). Following the completion of the dissection, a 38-Fr bougie was inserted into the stomach by anaesthetist (Figure 3). Under its guidance, the stomach was vertically sutured with two rows of stitches. The inner row was 10 to 13 full thicknesses non-absorbable interrupted stitches (2-0 Ethibond ${ }^{\mathrm{mx}}$; Ethicon) separated $2 \mathrm{~cm}$ a part $2 \mathrm{~cm}$ from angle of His to $3-4 \mathrm{~cm}$ from pylorus (Figure 4). The inner row sutures were reinforced with an outer layer continuous seromuscular stitches using the same suture material.

In the later cases of LGP we followed a slightly modified technique for plication as described by Skrekas et al. [4]. The inner row was done in a four-point fashion with two bites in the posterior gastric wall and two bites in the anterior one (interrupted mattress suture). The outer reinforcing layer was done in a continuous fashion (Figure 5).

Stomach tube distension test was performed by infusing normal saline $(150 \mathrm{cc})$ through the bougie to ensure the whole greater curve of stomach was totally invaginated by the two rows of sutures with no herniation between sutures. A tube drain was placed along the plicated greater curvature at the end of the procedure (Figure 6).

All patients were discharged once they can tolerate liquid diet. They were put on strict high-protein liquid diet for the first 2 weeks after surgery and puree diet from weeks 3 to 4 . Regular diet was gradually introduced thereafter. Proton pump inhibitor was given for 1 month after LGP. No anti-emetic was given after patient was discharged from the hospital. Patient was educated by dieticians to consume five to six

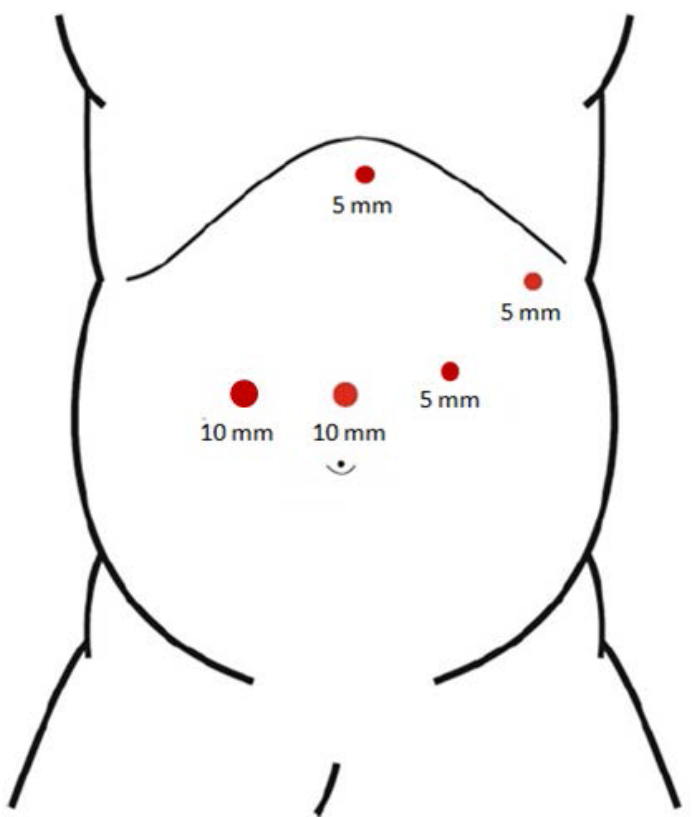

Figure 1: Trocar placement for LGP.

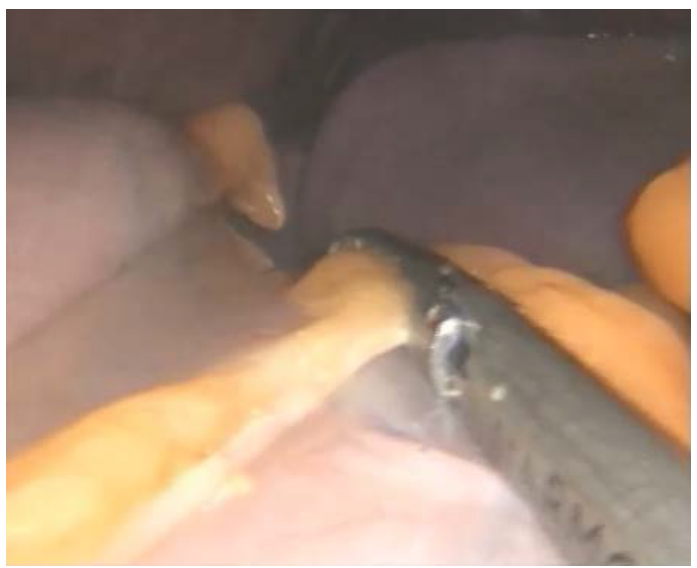

Figure 2: Division of gastrosplenic ligament away from gastric wall.

small meals each day and to avoid overeating and vomiting. Follow-up visits were scheduled at $1,3,6$, and 12 months postoperatively.

\section{Results}

Of the 58 patients who underwent LSG performed by the two authors (AE and NG), the data of 47 patients ( 9 males and 38 females) who had completed their 3- and 6-month postoperative follow-up (either outpatient visit or phone call) at the time of the study were further analyzed. The mean age was 36.8 years with a range of 22 to 54 years. Mean follow-up was 8.6 months. One patient had undergone intragastric balloon (BIB) prior to the LGP. The balloon was removed 6 months after its insertion and LGP was done one month after balloon removal. Mean preoperative weight was $109.4 \mathrm{~kg}$ with a mean BMI of 40.7 (range $36-54) \mathrm{kg} / \mathrm{m}^{2}$. At 3 months following LGP, mean weight loss was $24.6 \mathrm{~kg}$, and at 6 months $36.4 \mathrm{~kg}$. The mean \% EWL was 41.4 and 67.5 at 3 and 6 months respectively.

Patients with lower BMI $\left(<50 \mathrm{~kg} / \mathrm{m}^{2}\right)$ had better \%EWL at 3 months 
Citation: ElGeidie AA, ElHak NG (2013) Laparoscopic Gastric Plication in Obese Egyptian Patients: Technique and Initial Short-term Outcome. Surgery 3: 151. doi:10.4172/2161-1076.1000151

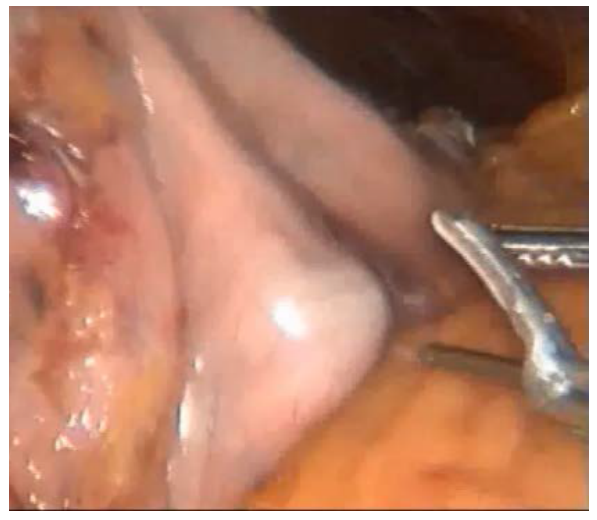

Figure 3: Calibration tube inside the stomach before plication.

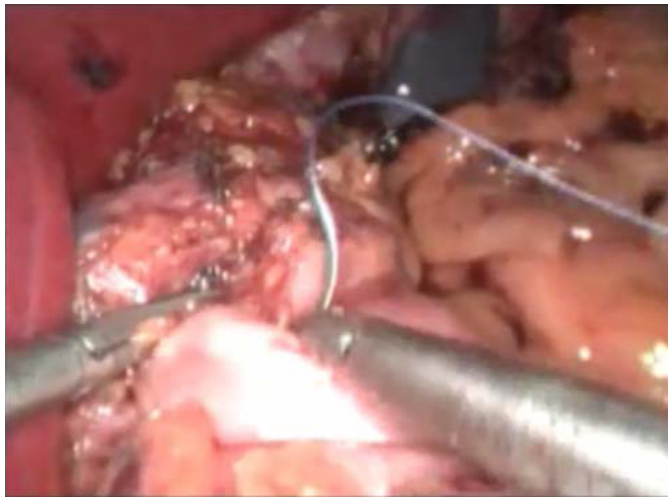

Figure 4: Plication stitches beginning about $2 \mathrm{~cm}$ below the angle of $\mathrm{His}$.

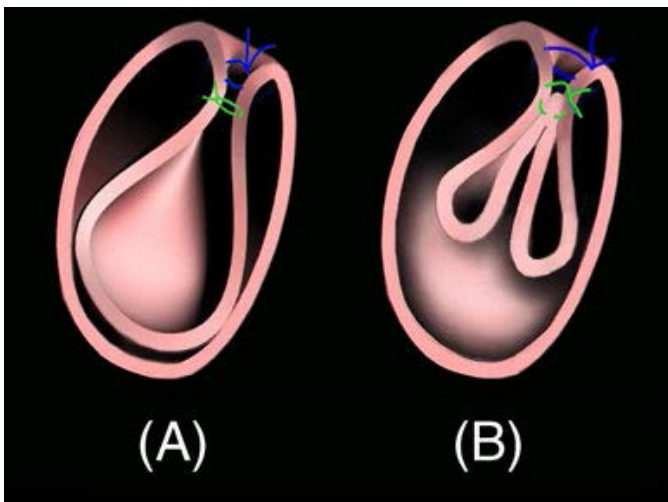

Figure 5: (A) Original technique used in early cases, (B) Modified technique used in later cases. Quoted from Skrekas [4].

( 38 vs. 33), but at 6 months the super-obese patients ( $\mathrm{n}=6, \mathrm{BMI}>50$ $\mathrm{kg} / \mathrm{m}^{2}$ ) achieved a better \%EWL (56 vs. 49). Mean BMI decreased to 32 and $31 \mathrm{~kg} / \mathrm{m}^{2}$ at 3 and 6 months respectively. Mean operative time was 85 (range 60-135) min. All the operations were completed laparoscopically. Mean hospital stay was 1.8 (range 1-7) days.

No mortality occurred in this study group. Two out of 58 patients (3.4\%) developed prolonged nausea, vomiting and sialorrhea for 5 and 7 days but subsided subsequently with antiemetics, proton pump inhibitors, and prokinetics. Those two patients were operated by the original technique while this complication did not occur in any patient operated by the four-bite method. No patient required reoperation or readmission. No patients showed regain of weight during the study. Inadequate weight loss $(\mathrm{EWL}<50 \%)$ and failure $(\mathrm{EWL}<30 \%)$ of weight loss had not been reported in any of our patients.

\section{Discussion}

Bariatric surgery is the most effective modality for long-term weight loss and for resolving the associated comorbidities [11]. Bariatric operations may be restrictive, malabsorptive, or both. Restrictive procedures are the most conservative bariatric surgery while malabsorptive method has longer effect on weight loss but the risk of late complications due to vitamin deficiency and anemia is noticeable [12]. LSG gained popularity in Egypt due to its technical simplicity and excellent weight loss.

However, complications have been seen in LSG owing to its long staple line with potential bleeding or even leakage. Moreover, the irreversible nature of LSG might be less attractive to some patients $[13,14]$

In an attempt to avoid the complications of restrictive procedures Talepbour and Amoli designed a new restrictive method after different stages of animal study named as Laparoscopic Gastric Plication (LGP) 12 years ago [1]. LGP bears many advantages but in a developing country like Egypt one of the most appealing features of this kind of surgery is its low cost due to elimination of the use of endostaplers. Therefore, morbidly obese patients who cannot afford the price of other costly bariatric surgeries like LSG would not be deprived the benefits of durable weight loss by surgery.

On the other hand, many LGP-related complications were described by other authors. Major complications such as acute gastric obstruction [3-7], gastric leaks and perforations ranging from minor leaks to full-blown gastric perforation [1,4-5], and even portosystemic thrombosis [4] had been reported but the most commonly reported side effect of LGP was nausea and vomiting. According to the review published by Abdelbaki et al. in 2012 including seven studies on gastric plication involving 307 patients [15] nausea and vomiting occurred in all studies ranging from mild to moderate. It usually resolved within 1 to 2 weeks with most of the cases did not require readmission and were managed with antiemetics and prokinetics. Twenty patients $(6.5$ $\%)$ were readmitted, of whom 14 (4.6\%) patients required reoperation. In this study nausea and vomiting occurred in most of our patients in the early postoperative days but only two patients had significant symptoms that mandated prolongation of hospital stay. In the late case we modified the technique of plication by using four-bite stitches

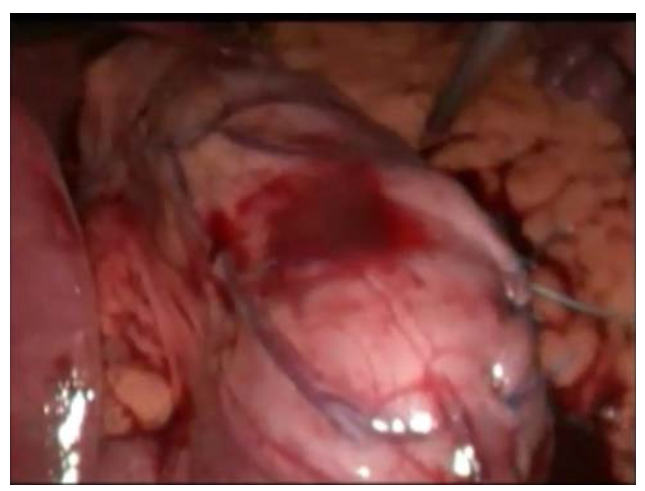

Figure 6: Final appearance of plicated stomach with calibration tube still inside the stomach. 
in the inner row of plication in order to avoid the creation of a large intraluminal septum of inverted gastric wall. This technique also decreases the pockets created in-between the gastric folds that might collect seroma and lead to gastric obstruction [4]. After adoption of this method we found marked improvement in the incidence and severity of post-LGP nausea and vomiting. We are already running a prospective randomized study comparing both techniques and waiting for the results.

Unlike LSG, the most obvious deficit in LGP is lack of standardization among different surgeons. Like most series, a 38-French calibration device was used in this study to adjust the volume of stomach. Moreover, the calibration tube eliminates the potential risk of suturing the lesser curvature of the stomach.

Most authors starting dissection 3-4 $\mathrm{cm}$ proximal to the pylorus all the way up to the angle of His exposing the left crus of diaphragm $[2,4]$ but in our cases we stopped $2 \mathrm{~cm}$ below the angle of His without dissection of the left diaphragmatic crus or the esophageal pad of fat. We believe that starting plication few centimeters below the angle of His avoids the formation of an obstructing valve below cardioesophageal junction thus decreasing the risk of obstruction.

Our study includes a small number of patients with a short follow up period. Our initial results were promising. Most patients showed a substantial body weight loss after surgery with a comparable early weight loss results to other restrictive procedures. More importantly, we noticed that the procedure is very safe with minimal morbidities. No major complications had been reported in any of our patients.

We believe that LGP is suitable for Egyptian patients and more readily accepted in our locality than in western countries due to many reasons. First, the most popular procedures in Egypt, namely LAGB and LSG, have their own specific problems and disadvantages. LAGB has recently been losing popularity and is slowly substituted by LSG [16]. LAGB has been shown to be less effective among common bariatric procedures with a high rate of reoperation, a need for frequent postoperative adjustments, poor quality of eating, and risk of band slippage and band erosion $[2,17,18]$. Many patients in Egypt will not opt for LAGB, which requires strict compliance to follow-up and foreign body implantation.

On the other hand, although sleeve gastrectomy is now gaining popularity worldwide, it may not be immediately accepted by all groups of patients. Most of our patients prefer less risky and reversible procedures. Hence, LGP will become a good alternative to those who cannot accept even the slight chance of staple leakage and bleeding that would occur with LSG. More importantly, LSG is an expensive operation because of the need for endostaplers $[2,17,19]$.

Several questions need to be addressed to further establish the role of LGP. We definitely require more prolonged follow-up to assess the long-term results of weight loss. Randomized trials comparing LGP with other procedures are warranted. In addition, possibility of reversing, converting or combining LGP to other operations mandates more investigation. A recently published Egyptian study concluded that LGP is inferior as a restrictive procedure for resolution of type 2 diabetes [20] otherwise we have no enough data regarding the impact of LGP on diabetes and metabolic syndrome associated with obesity and the metabolic and hormonal effect of LGP is uncertain. As a result, extreme caution is required when LGP is applied to these groups of patients at the present moment. LGP is a technically demanding procedure and the technique requires further standardization before it can be widely accepted and promoted.

\section{References}

1. Talebpour M, Amoli BS (2007) Laparoscopic total gastric vertical plication in morbid obesity. J Laparoendosc Adv Surg Tech A 17: 793-798.

2. Ramos A, Galvao Neto M, Galvao M, Evangelista LF, Campos JM, et al (2010) Laparoscopic greater curvature plication: initial results of an alternative restrictive bariatric procedure. Obes Surg 20: 913-918.

3. Brethauer SA, Harris JL, Kroh M, Schauer PR (2011) Laparoscopic gastric plication for treatment of severe obesity. Surg Obes Relat Dis 7: 15-22.

4. Skrekas G, Antiochos K, Stafyla VK (2011) Laparoscopic gastric greater curvature plication: results and complications in a series of 135 patients. Obes Surg 21: 1657-1663.

5. Watkins BM (2012) Gastric compartment syndrome: an unusual complication of gastric plication surgery. Surg Obes Relat Dis 8: e80-81.

6. Tsang A, Jain V (2012) Pitfalls of bariatric tourism: a complication of gastric plication. Surg Obes Relat Dis 8: e77-79.

7. Pujol Gebelli J, García Ruiz de Gordejuela A, Casajoana Badía A, Secanella Medayo L, Vicens Morton A, et al. (2011) [Laparoscopic Gastric Plication: a new surgery for the treatment of morbid obesity]. Cir Esp 89: 356-361.

8. Fried M, Dolezalova K, Buchwald JN, McGlennon TW, Sramkova $P$ et al. (2012) Laparoscopic greater curvature plication (LGCP) for treatment of morbid obesity in a series of 244 patients. Obes Surg 22: 1298-1307.

9. Deitel M, Greenstein RJ (2003) Recommendations for reporting weight loss Obes Surg 13: 159-160.

10. (1992) Gastrointestinal surgery for severe obesity: National Institutes of Health Consensus Development Conference Statement. Am J Clin Nutr 55: 615S-619S.

11. Buchwald H, Avidor $Y$, Braunwald E, Jensen MD, Pories W, et al. (2004) Bariatric surgery: a systematic review and meta-analysis. JAMA 292: 17241737.

12. Crea N, Pata G, Di Betta E, Greco F, Casella C, et al. (2011) Long-term results of biliopancreatic diversion with or without gastric preservation for morbid obesity. Obes Surg 21: 139-145.

13. Ocón Bretón J, Pérez Naranjo S, Gimeno Laborda S, Benito Ruesca P, García Hernández R (2005) [Effectiveness and complications of bariatric surgery in the treatment of morbid obesity]. Nutr Hosp 20: 409-414.

14. Baltasar A, Bou R, Bengochea M, Serra C, Cipagauta L (2007) Use of a Roux limb to correct esophagogastric junction fistulas after sleeve gastrectomy. Obes Surg 17: 1408-1410.

15. Abdelbaki TN, Huang CK, Ramos A, Neto MG, Talebpour M, et al. (2012) Gastric plication for morbid obesity: a systematic review. Obes Surg 22: 1633 1639.

16. Wong SK, Mui WL, Ng EK (2009) Development of bariatric surgery: the effectiveness of a multi-disciplinary weight management programme in Hong Kong. Ann Acad Med Singapore 38: 9-6.

17. Picot J, Jones J, Colquitt JL, Gospodarevskaya E, Loveman E, et al. (2009) The clinical effectiveness and cost-effectiveness of bariatric (weight loss) surgery for obesity: a systematic review and economic evaluation. Health Technol Assess 13: 1-190, 215-357, iii-iv.

18. Nocca D, Frering V, Gallix B, de Seguin des Hons C, Noël P, et al. (2005) Migration of adjustable gastric banding from a cohort study of 4236 patients. Surg Endosc 19: 947-950.

19. Lalor PF, Tucker ON, Szomstein S, Rosenthal RJ (2008) Complications after laparoscopic sleeve gastrectomy. Surg Obes Relat Dis 4: 33-38.

20. Taha O (2012) Efficacy of laparoscopic greater curvature plication for weight loss and type 2 diabetes: 1-year follow-up. Obes Surg 22: 1629-1632.

This article was originally published in a special issue, Treatment of Morbid Obesity handled by Editor. Dr. Mark Antal, University of Szeged, USA 